\title{
Low expression of microRNA-328 can predict sepsis and alleviate sepsis-induced cardiac dysfunction and inflammatory response
}

\author{
Bin Sun (ii) ${ }^{1}$, Chunye Luan (ii) ${ }^{1}$, Lisha Guo (ii) ${ }^{1}$, Bing Zhang ${ }^{1}{ }^{1}$, and Yufang Liu (i) ${ }^{2}$ \\ ${ }^{1}$ Department of Emergency, Binzhou Medical University Hospital, Binzhou, Shandong, China \\ ${ }^{2}$ Department of Gynaecology and Obstetrics, Binzhou Medical University Hospital, Binzhou, Shandong, China
}

\begin{abstract}
Sepsis often leads to cardiac dysfunction and inflammation. This study investigated the clinical value of microRNA-328 (miR328 ) in sepsis and its role in cardiac dysfunction and inflammation caused by sepsis. The expression level of miR-328 in the serum of the subjects was detected by qRT-PCR. Receiver operating characteristic (ROC) curve measured the diagnostic value of miR-328 in sepsis. Rat sepsis model was established to detect left ventricular systolic pressure (LVSP), left ventricular enddiastolic pressure (LVEDP), and maximal rate of increase/decrease of left ventricular pressure ( $\pm \mathrm{dp} / \mathrm{dt}_{\text {max }}$ ). Myocardial injury markers serum cardiac troponin I (cTnl), myocardial kinase isoenzyme (CK-MB), and inflammatory factors were detected by enzyme-linked immunosorbent assay (ELISA). miR-328 expression was assessed in serum of sepsis patients and in rat models of sepsis. The AUC of ROC curve was 0.926 , sensitivity $87.60 \%$, and specificity $86.36 \%$. Compared with the sham group, LVSP and $+\mathrm{dp} / \mathrm{dt}_{\max }$ were decreased in the rat model of sepsis. LVEDP, $-\mathrm{dp} / \mathrm{dt}_{\max }, \mathrm{cTnl}, \mathrm{CK}-\mathrm{MB}$, tumor necrosis factor- $\alpha$, interleukin (IL)-6, and IL-1 $\beta$ were upregulated in the rat model of sepsis. The low expression of miR-328 reversed these indicators. miR-328 is a diagnostic marker for patients with sepsis, and decreasing the expression level of miR-328 can ameliorate cardiac dysfunction and cardiac inflammation in sepsis.
\end{abstract}

Key words: miR-328; Sepsis; Cardiac dysfunction; Inflammatory response; Diagnosis

\section{Introduction}

Sepsis has been identified as a systemic inflammatory response to infection or injury (1). About $50 \%$ of sepsis patients require intensive care unit treatment $(2,3)$, leading to high morbidity and mortality $(4,5)$. The annual incidence of severe sepsis is increasing, with a recent report showing 132 cases of patients per 100,000 people, a mortality rate of nearly $50 \%$, and the high financial burden of $\$ 50,000$ per patient for nursing care $(6,7)$. Cardiac dysfunction is one of the common complications of sepsis-induced death $(8,9)$. Myocardial injury and myocardial depression are the most common cardiac dysfunctions caused by sepsis (10). In the diagnosis of sepsis, the analysis of blood microbial culture is the gold standard, but it takes a long time and an early positive rate is low (11). As a systemic inflammatory response, inflammation-related serum C-reactive protein (CRP), procalcitonin (PTC), and interleukin (IL-6) have been used in the diagnosis of sepsis $(12,13)$, but their specificity and sensitivity are limited by different conditions. Therefore, new biomarkers are urgently needed for the early diagnosis and accurate assessment of sepsis patients.
MicroRNA are endogenous, non-coding small RNA molecules consisting of 22 nucleotides. Abnormal expression of miRNA has been detected in a variety of human disease states including sepsis, cardiovascular disease, inflammation, and tumor. miR-328 is a miRNA located on chromosome 16q22.1 and is involved in various diseases including lung cancer (14), osteosarcoma (15), nasopharyngeal (16), myocardial infarction (17), and chronic leukemia (18). A recent study indicated that miR-328 can promote myocardial fibrosis through paracrine regulation of cardiomyocytes (19), resulting in cardiac dysfunction. Additionally, miR-328 promotes cardiac fibrosis by stimulating the tumor growth factor- $\beta 1$ signaling pathway to promote collagen production (20). All evidence suggests a crucial role for miR-328 in myocardial function. Furthermore, miR-328 is also reported to be significantly upregulated in Kawasaki disease with acute systemic vasculitis (21), indicating its important role in inflammatory response. However, the role of miR-328 in sepsis and sepsisinduced cardiac dysfunction and inflammation is unknown. 
This study investigated the expression level and clinical role of miR-328 in patients with sepsis. In addition, the role of miR-328 in cardiac dysfunction and inflammation caused by sepsis was studied.

\section{Material and Methods}

\section{Subject data and blood samples}

Patients with sepsis who were admitted to the intensive care unit of Binzhou Medical University Hospital from January 2016 to December 2017 were recruited. The inclusion criteria for this study were based on consensus definition of the American College of Chest Physicians/ Intensive Care Medicine, and the results of blood microbial culture were used to diagnose sepsis in patients (22). Patients with the following conditions were excluded: 1) receiving immunosuppressive drugs within the last 3 months; 2) pregnancy or lactation; 3) severe chronic diseases such as heart, liver, kidney, and lung; 4) suffering from solid cancer or hematological malignancies; 5) human immunodeficiency virus infection; and 6) immunodeficiency. A total of 110 patients were studied after screening. At the same time, 89 healthy volunteers of similar age and gender and no systemic inflammation, tumor history, or heart and kidney dysfunction were recruited from the health check-up center. Organ dysfunction (SOFA) score (23) and acute physiology and chronic health (APACHE-II) score (24) were applied in patients with sepsis within $24 \mathrm{~h}$ of initial admission to the ICU, and blood samples were collected from patients for testing. Clinical information such as age, gender, body mass index (BMI), serum creatinine (Scr), white blood cells (WBC), albumin, PTC, and CRP were recorded.

The study was approved by the Medical Ethics Committee of Binzhou Medical University Hospital and all participants or family members signed the consent form. All patients were treated according to current guidelines for the treatment of sepsis (survival of sepsis) and specific guidelines from relevant committees.

\section{Animals and animal model of sepsis}

Forty adult male Sprague-Dawley rats weighing 250$300 \mathrm{~g}$ were purchased from Shanghai Animal Center (China). Rats were housed at $22-23^{\circ} \mathrm{C}, 50 \%$ humidity, with a 12/12 light and dark schedule for at least 5 days for adaptation before conducting experiments. All animal experiments were performed in accordance with the animal care and use guidelines of the Binzhou Medical University Hospital Ethics Committee. Establishment of the model of cecal ligation and perforation (CLP) sepsis in rats was done in a sterile environment. First, the rats were anesthetized with $50 \mathrm{mg} / \mathrm{kg}$ pentobarbital sodium and then treated with $75 \%$ ethanol for intraperitoneal disinfection. After anesthesia, the rats were incised $2 \mathrm{~cm}$ in the middle of the lower abdomen to expose the cecum, which was ligated. A sterile needle 18 was used to puncture the cecum twice, and the cecum was put back into the abdominal cavity after gently squeezing out the feces. Finally the abdominal incision was sutured. The sham group had the same operation except with no ligation and puncture. After the operation, the rats were placed on a constant temperature heating pad, and the body temperature was maintained at $36-37^{\circ} \mathrm{C}$.

\section{Groups of animals}

Rats were divided into 4 groups with 10 rats in each group: sham group, CLP group, miR-328 negative control group (miR-328 NC), and miR-328 antagomir group. In the sham group, $1 \mathrm{~mL}$ of normal saline was injected into the tail vein $24 \mathrm{~h}$ before surgery. In the CLP group, $1 \mathrm{~mL}$ of normal saline was injected into the tail vein of CLP-treated rats $24 \mathrm{~h}$ before surgery. The miR-328 NC group was injected with $10 \mu \mathrm{g} \mathrm{miR} \mathrm{NC}$ sequence into the tail vein of CLP-treated rats $24 \mathrm{~h}$ before surgery. The miR-328 antagomir group was injected with $10 \mu \mathrm{g}$ miR antagomir sequence into the tail vein of CLP-treated rats $24 \mathrm{~h}$ before surgery. After successful modeling, at least 8 individuals survived in each group. miR-328 NC and miR-328 antagomir were synthesized by GenePharma (China).

\section{Cardiac function measurement and blood cytokines assessments}

Rats were tested for cardiac function after surgery. After the rats were anesthetized, a catheter was inserted into the left ventricle through the right common artery. Analyses of changes in rat hemodynamic parameters, such as left ventricular end-diastolic pressure (LVEDP), left ventricular systolic pressure (LVSP), the maximum rate of increase in left ventricular blood pressure $\left(+\mathrm{dp} / \mathrm{dt}_{\max }\right)$, and the maximum rate of decrease in left ventricular blood pressure $\left(-\mathrm{dp} / \mathrm{dt}_{\max }\right)$, were performed using the MFLab 3.01 software in the FDP-1 HRV \& BRS analysis system. After cardiac function was measured, $5 \mathrm{~mL}$ of inferior vena cava blood was placed into a test tube with added anticoagulant, and serum was collected by centrifugation at $2,500 \mathrm{~g}$ for $15 \mathrm{~min}$ at room temperature for enzymelinked immunoreactivity assay (ELISA). ELISA kit (Abcam, UK) was used for the detection of tumor necrosis factor $\alpha$ (TNF- $\alpha$ ), interleukin $1 \beta$ (IL-1 $\beta)$, interleukin 6 (IL-6), and biomarkers of myocardial injury in rat serum. Expression levels of creatine kinase isoenzyme (CK-MB) and troponin (cTnl) were read at $450 \mathrm{~nm}$ absorbance.

\section{Quantitative real-time polymerase chain reaction (qRT-PCR)}

After the end of the rat experiment, each group of rats was sacrificed by cervical dislocation. Total RNA in heart tissue was isolated using TRIZOL. RNA extraction reagent (Invitrogen, USA) was used, and total RNA reverse transcription was performed using the PrimerScript Real-time 
reagent kit (TAKARA, Japan). Finally, qRT-PCR was detected by ABI PRISM 7000 (Applied Biosystems, USA) by SYBR Premix Ex Taq TM II reagent. Using U6 as an internal reference, the relative expression of miR328 was calculated by $2^{-\Delta \Delta C t}$.

\section{Statistical analysis}

Data are reported as means $\pm \mathrm{SD}$, and Student's $t$-test was used to compare differences between two groups. The receiver operating characteristic (ROC) curve was drawn to evaluate the diagnostic value of miR-328 level in patients with sepsis, and the area under the curve (AUC) was calculated. Spearman correlation analysis examined the relationship between miR-328 expression and clinical characteristics of patients. Statistical analysis was performed using SPSS 19.0 software and GraphPad Prism 7.0 software (USA). $\mathrm{P}<0.05$ was considered to be statistically significant.

\section{Results}

\section{Clinical characteristics of study subjects}

One hundred and ten sepsis patients (69 males/41 females, mean age $54.85 \pm 4.99$ ) and 89 healthy controls (58 males/31 females, mean age $55.88 \pm 4.90$ ) were enrolled. The demographic and clinical characteristics of the two groups of subjects are shown in Table 1. There was no significant difference in age $(P<0.150)$, gender $(P<0.768)$, and BMI $(P<0.258)$ between the healthy control group and the sepsis patients group, but significant differences in Scr, WBC, CRP, and PTC were observed $(P<0.001)$. At the same time, APACHE II $(12.09 \pm 2.30)$ and SOFA $(4.8 \pm 1.25)$ scores of sepsis patients were significantly higher than those of the healthy control group.

\section{Serum level of miR-328 in sepsis patients}

The expression level of miR-328 in patients with sepsis was significantly higher than that in the healthy control group $(P<0.001)$. Therefore, it was speculated that miR328 played a crucial role in sepsis (Figure 1).

\section{Correlation of miR-328 expression with clinicopathological features of sepsis patients}

The expression of miR-328 was significantly positively correlated with Scr, WBC, CRP, PTC, APACHE II score, and SOFA score $(P<0.01)$, but had no significant correlation with age, gender, $\mathrm{BMI}$, and albumin $(P>0.05)$. The experimental results showed that the expression level of miR-328 was significantly positively correlated with the condition of sepsis (Table 2).

\section{Diagnostic value of $\mathrm{miR}-328$ in sepsis}

The AUC of miR-328 was 0.926 , the cut-off value was 0.305 , the sensitivity was $87.60 \%$, and the specificity was $86.36 \%$. The ROC curve showed that miR-328 has a good diagnostic value for sepsis (Figure 2).

\section{Effect of miR-328 on cardiac dysfunction in sepsis rat model}

The expression of miR-328 was significantly up-regulated in rat tissues and serum after CLP modeling, but the high expression was reversed when miR-328 antagomir was injected $(P<0.01$, Figure $3 A$ and $B)$. In addition, compared with the sham group, LVSP and $+\mathrm{dp} / \mathrm{dt}_{\max }$ decreased significantly in the CLP group, while the levels of $-d p / \mathrm{dt}_{\max }$, LVEDP, cTnl, and CK-MB were significantly increased $(P<0.01$, Figure $3 C-G)$. The experimental results showed that myocardial dysfunction occurred in the rat model of sepsis. However, when miR-328 antagomir was injected,

Table 1. Comparison of the baseline data between the healthy control group and the sepsis group of patients.

\begin{tabular}{lccc}
\hline Parameters & Healthy $(\mathrm{n}=89)$ & Sepsis $(\mathrm{n}=110)$ & P value \\
\hline Age (years) & $55.88 \pm 4.91$ & $54.85 \pm 4.99$ & 0.150 \\
Gender (male/female) & $58 / 31$ & $69 / 41$ & $0.723^{*}$ \\
BMl $\left(\mathrm{kg} / \mathrm{m}^{2}\right)$ & $20.83 \pm 1.19$ & $20.63 \pm 1.21$ & 0.258 \\
Scr $(\mathrm{mg} / \mathrm{dL})$ & $0.97 \pm 0.21$ & $1.61 \pm 0.188$ & $<0.001$ \\
Albumin $(\mathrm{g} / \mathrm{L})$ & $40.76 \pm 3.07$ & $30.11 \pm 5.50$ & $<0.001$ \\
WBC $\left(\times 10^{9} / \mathrm{L}\right)$ & $7.48 \pm 1.56$ & $18.43 \pm 3.19$ & $<0.001$ \\
CRP $(\mathrm{mg} / \mathrm{L})$ & $7.10 \pm 2.07$ & $69.12 \pm 15.07$ & $<0.001$ \\
PCT $(\mathrm{ng} / \mathrm{mL})$ & $0.06 \pm 0.02$ & $11.54 \pm 2.62$ & $<0.001$ \\
APACHE II score & - & $12.09 \pm 2.30$ & - \\
SOFA score & - & $4.8 \pm 1.25$ & - \\
\hline
\end{tabular}

BMI: body mass index; Scr: serum creatinine; WBC: white blood cells; CRP: Creactive protein; PCT: procalcitonin; APACHE: acute physiology and chronic health evaluation; SOFA: sequential organ failure assessment. Data are reported as means \pm SD (Student's $t$-test or *chi-squared test). 


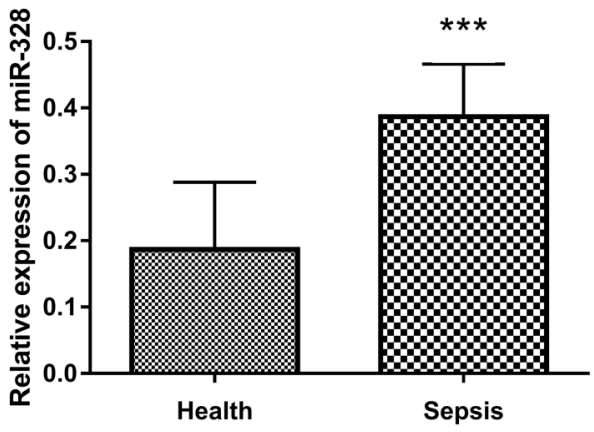

Figure 1. The expression level of miR-328 in the serum of sepsis patients and healthy controls was detected by qQT-PCR. Data are reported as means $\pm \mathrm{SD}$. ${ }^{* * *} \mathrm{P}<0.001$ (Student's $t$-test).

Table 2. Correlation of miR-328 relative expression with clinical characteristics of sepsis patients.

\begin{tabular}{lcc}
\hline Parameters & \multicolumn{2}{c}{ MiR-328 expression } \\
\cline { 2 - 3 } & P value & $\begin{array}{c}\text { Correlation } \\
\text { coefficient }(r)\end{array}$ \\
\hline Age & 0.223 & -0.117 \\
Gender & 0.665 & -0.042 \\
BMl & 0.253 & -0.110 \\
Scr & 0.009 & 0.248 \\
Albumin & 0.099 & -0.158 \\
WBC & $<0.001$ & 0.395 \\
CRP & $<0.001$ & 0.486 \\
PCT & $<0.001$ & 0.425 \\
APACHE II score & $<0.001$ & 0.577 \\
SOFA score & $<0.001$ & 0.552 \\
\hline
\end{tabular}

BMI: body mass index; Scr: serum creatinine; WBC: white blood cell; CRP: C-reactive protein; PCT: procalcitonin; APACHE: acute physiology and chronic health evaluation; SOFA: sequential organ failure assessment. Spearman correlation analysis.

myocardial dysfunction in sepsis rats was reversed, LVSP and $+d p / d t_{\text {max }}$ were significantly increased, levels of $-\mathrm{dp} / \mathrm{dt}_{\max }$, LVEDP, cTnl, and CK-MB were significantly decreased $(P<0.001$, Figure $3 C-G)$. Therefore, it was determined by the rat sepsis model that miR-328 may be a potential mechanism for the regulation of myocardial function in sepsis.

\section{Effect of miR-328 on inflammatory responses in sepsis rat model}

The expression levels of TNF- $\alpha$, IL- 6 , and IL- $1 \beta$ were significantly increased in the CLP group compared with the sham group, indicating that sepsis promoted inflammation. However, injection of miR-328 antagomir reduced the inflammatory response, resulting in decreased levels of TNF- $\alpha$, IL- 6 , and IL- $1 \beta$ expression $(P<0.01$, Figure $4 \mathrm{~A}-\mathrm{C})$. The results of this study demonstrated that

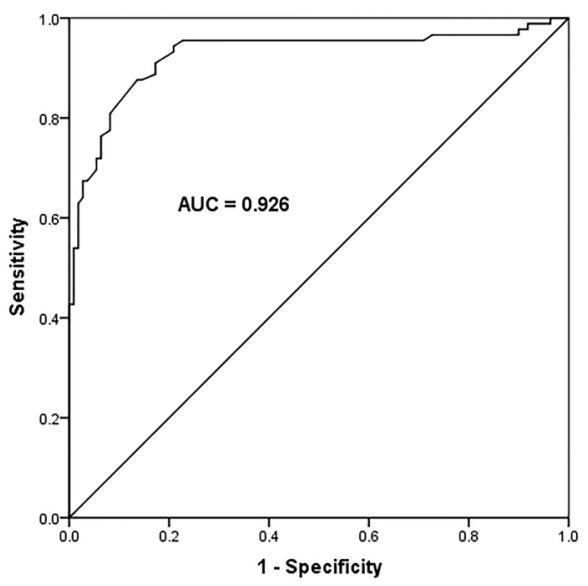

Figure 2. The receiver operating characteristic curve was used to analyze the diagnostic value of miR-328 in sepsis. The area under the curve (AUC) was 0.926 , the sensitivity was $87.60 \%$, and the specificity was $86.36 \%$.

miR-328 affects changes in the inflammatory response in sepsis.

\section{Discussion}

Sepsis is a common cause of death in hospitalized patients worldwide and is still a huge challenge in clinical medicine due to its high mortality and morbidity. The current diagnostic markers CRP, IL-6, PTC, and other biomarkers are more sensitive but less specific, so new diagnostic markers must be investigated to help determine the most appropriate treatment to reduce patient mortality $(25,26)$.

miRNAs are part of a complex regulatory network in the regulation of physiological and pathological processes of gene expression. Abnormal expression of miRNA is not only confirmed in development, aging, and cell death $(27,28)$, but is also found in complex diseases such as infection, inflammation, and sepsis (29-31). Previous studies have reported that miR-328 is abnormally expressed in a variety of diseases and can be used as a marker for diagnosis and prognosis. miR-328 is significantly down-regulated in gliomas and can inhibit cell proliferation and invasion, being a good prognostic marker for glioma (32). High expression of miR-328 in peripheral blood is an effective marker for early diagnosis of non-small cell lung cancer (33). In our study, we have demonstrated for the first time that miR-328 was highly expressed in patients with sepsis, and the ROC curve confirmed that miR-328 was an effective diagnostic marker for sepsis.

Previous studies have confirmed that cardiac dysfunction as a common complication of sepsis can seriously affect a patient's health and is associated with increased mortality (34). LVSP and $+\mathrm{dp} / \mathrm{dt}_{\max }$ are key indicators of 
A

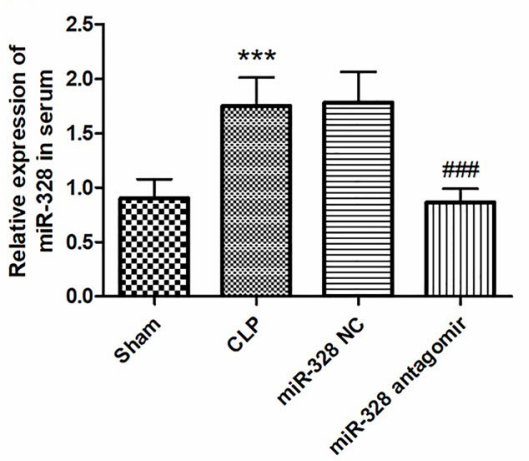

C

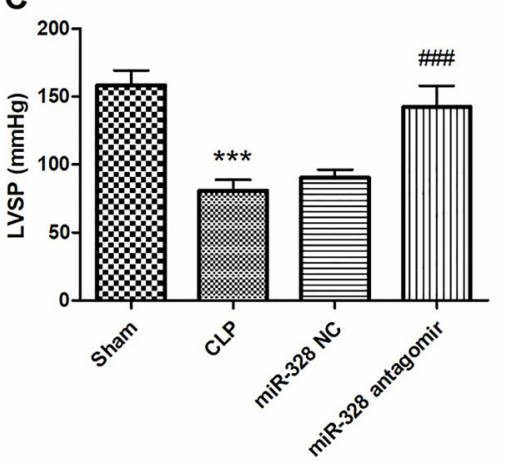

E

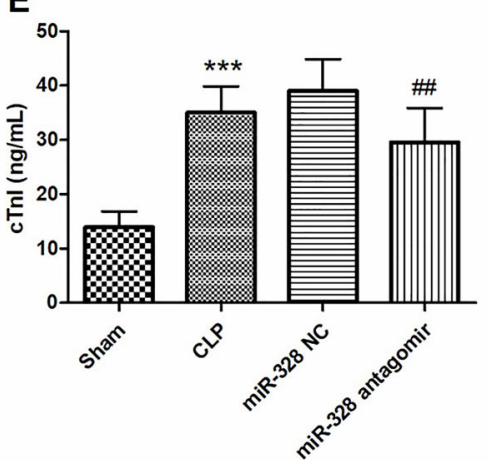

B

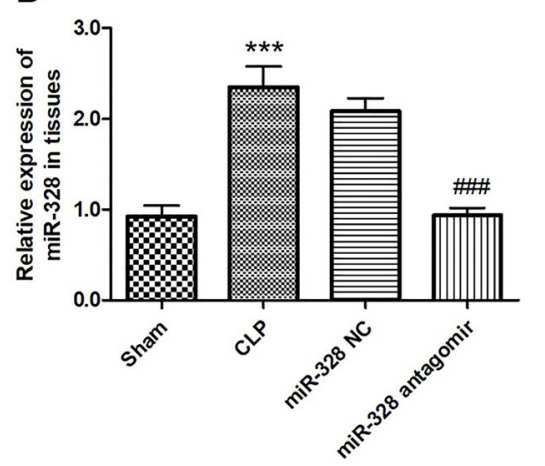

D

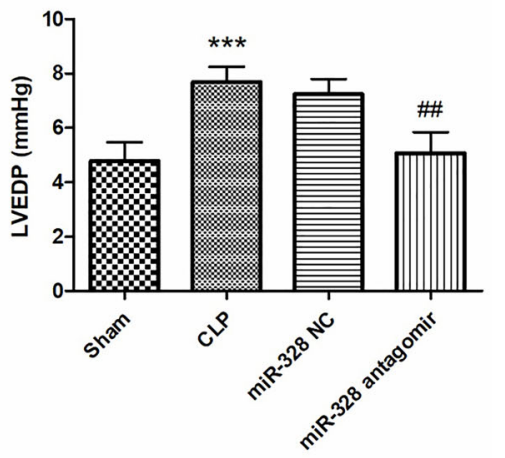

F

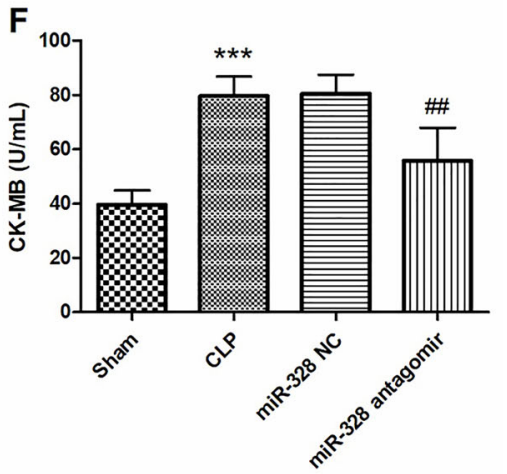

Sham

CLP

miR-328 NC

III miR-328 antagomir
G

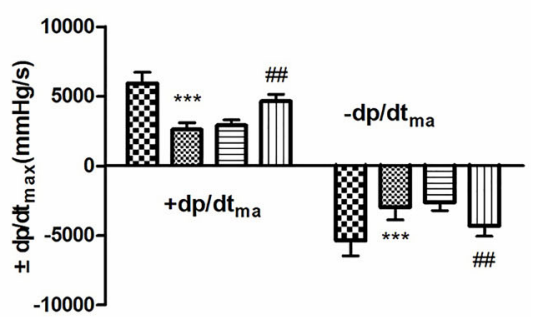

Figure 3. Effect of miR-328 on cardiac dysfunction in a rat model of sepsis. A and B, Changes in expression levels of miR-328 in serum and myocardial tissue after establishment of a rat model of sepsis and after injection of miR-328 antagomir. C-G, Modeling of sepsis in rats and changes in cardiac hemodynamics and serum myocardial injury after miR-328 antagomir injection. Data are reported as means \pm SD. ${ }^{* * *} \mathrm{P}<0.001$, compared with sham group; ${ }^{\# \#} \mathrm{P}<0.01,{ }^{\# \#} \mathrm{P}<0.001$, compared with CLP group (Student's $t$-test). CLP: cecal ligation and perforation; NC: negative control; LVSP: left ventricular systolic pressure; LVDEP: left ventricular end-diastolic pressure; cTnl: serum cardiac troponin I; CK-MB: myocardial kinase isoenzyme; $\pm \mathrm{dp}_{\mathrm{dt}}$ max : maximum rate of increase/decrease in left ventricular blood pressure.

myocardial contractility, and LVEDP and $-\mathrm{dp} / \mathrm{dt}_{\max }$ are valuable indicators of myocardial relaxation (35). In our study, the levels of LVSP and $+\mathrm{dp} / \mathrm{dt}_{\max }$ were significantly reduced in the CLP group, while the levels of LVEDP and $-\mathrm{dp} / \mathrm{dt}_{\max }$ were significantly elevated, indicating that myocardial contraction and diastolic dysfunction were caused by sepsis. cTnl and CK-MB are specific biomarkers for myocardial injury (36). In our study, the levels of cTnl and
CK-MB in the CLP group were significantly elevated, and experiments confirmed that sepsis can cause myocardial damage. This was consistent with previous findings that sepsis can lead to cardiac dysfunction. A study by Zhao et al. (19) in 2018 found that cardiomyocyte-derived miR-328 can promote myocardial fibrosis by paracrine regulation of adjacent fibroblasts. In 2016, Du et al. (20) confirmed that miR-328 was up-regulated in the marginal 
A

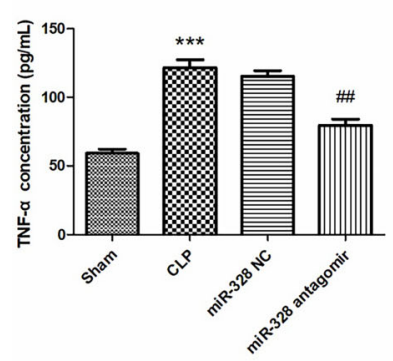

B

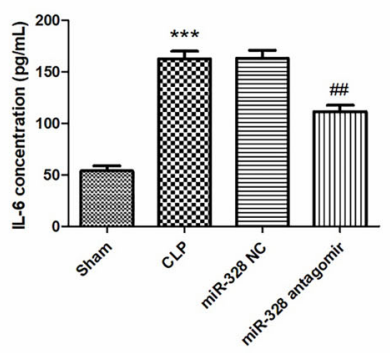

C

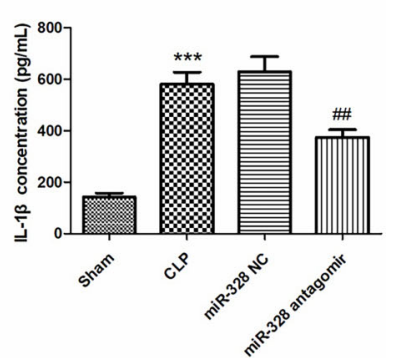

Figure 4. Changes in inflammatory factors in the sepsis rat model by cecal ligation and perforation (CLP) and after injection of miR-328 antagomir. Data are reported as means $\pm S D$. ${ }^{* * *} \mathrm{P}<0.001$, compared with sham group; ${ }^{\# \#} \mathrm{P}<0.01$, compared with $\mathrm{CLP}$ group (Student's $t$-test). NC: negative control. TNF- $\alpha$ : tumor necrosis factor- $\alpha$; IL: interleukin.

zone of myocardial infarction in mice, confirming that miR328 is an effective miRNA that promotes fibrosis. In the present study, it was confirmed that down-regulation of miR-328 expression level alleviated cardiac dysfunction caused by sepsis, such as myocardial contractile function inhibition, myocardial diastolic function promotion, and myocardial injury. The experimental results confirmed that in sepsis, the down-regulation of miR-328 helped to alleviate cardiac dysfunction and provided protection for the myocardium.

Sepsis is a systemic inflammatory response syndrome that is the leading cause of cardiac dysfunction (37). We have demonstrated that inhibition of miR-328 can ameliorate cardiac dysfunction in sepsis, and it has been reported that miR-328 is associated with inflammatory response, so we further speculated whether miR-328 can affect the inflammatory response in sepsis. Zhang et al. (21) confirmed

\section{References}

1. Venet F, Rimmele T, Monneret G. Management of sepsisinduced immunosuppression. Crit Care Clin 2018; 34: 97-106, doi: 10.1016/j.ccc.2017.08.007.

2. Angus DC, Linde-Zwirble WT, Lidicker J, Clermont G, Carcillo J, Pinsky MR. Epidemiology of severe sepsis in the United States: analysis of incidence, outcome, and associated costs of care. Crit Care Med 2001; 29: 13031310, doi: 10.1097/00003246-200107000-00002.

3. Lagu T, Rothberg MB, Shieh MS, Pekow PS, Steingrub JS, Lindenauer PK. Hospitalizations, costs, and outcomes of severe sepsis in the United States 2003 to 2007. Crit Care Med 2012; 40: 754-761, doi: 10.1097/CCM.0b013e318 $232 \mathrm{db} 65$.

4. Lago AF, de Oliveira AS, de Souza HCD, da Silva JS, BasileFilho A, Gastaldi AC. The effects of physical therapy with neuromuscular electrical stimulation in patients with septic shock: study protocol for a randomized cross-over design. Medicine 2018; 97: e9736, doi: 10.1097/MD.0000000000009736.

5. Venkatesh B, Finfer S, Cohen J, Rajbhandari D, Arabi $Y$, Bellomo $\mathrm{R}$, et al. Adjunctive glucocorticoid therapy in patients with septic shock. N Engl J Med 2018; 378: 797808, doi: 10.1056/NEJMoa1705835. that miR-328 in the serum of patients with Kawasaki disease caused by systemic vascular inflammation is significantly up-regulated and can be used as a biomarker for diagnosis and prediction. Meanwhile, recent studies have reported that five miRNAs, including miR-328, play a regulatory role in non-small cell lung cancer by affecting inflammation-related signaling pathways (38). Consistently, in our experiment, inflammatory cytokines were significantly up-regulated in the CLP group, confirming that sepsis can promote the occurrence of inflammatory response. The decrease of $\mathrm{miR}-328$ reduced the expression of inflammatory factors in sepsis.

In conclusion, miR-328 was highly expressed in the serum of patients with sepsis, and down-regulation of miR328 can alleviate cardiac dysfunction and inflammatory response in sepsis. miR-328 can be used as a diagnostic marker for sepsis for early diagnosis and treatment.

6. Chalfin DB, Holbein ME, Fein AM, Carlon GC. Costeffectiveness of monoclonal antibodies to gram-negative endotoxin in the treatment of gram-negative sepsis in ICU patients. JAMA 1993; 269: 249-254, doi: 10.1001/jama. 1993.03500020083037.

7. Dombrovskiy VY, Martin AA, Sunderram J, Paz HL. Rapid increase in hospitalization and mortality rates for severe sepsis in the United States: a trend analysis from 1993 to 2003. Crit Care Med 2007; 35: 1244-1250, doi: 10.1097/01. CCM.0000261890.41311.E9.

8. Yende S, Linde-Zwirble W, Mayr F, Weissfeld LA, Reis S, Angus DC. Risk of cardiovascular events in survivors of severe sepsis. Am J Resp Crit Care Med 2014; 189: 10651074, doi: 10.1164/rccm.201307-13210C.

9. Sato R, Kuriyama A, Takada T, Nasu M, Luthe SK. Prevalence and risk factors of sepsis-induced cardiomyopathy: a retrospective cohort study. Medicine 2016; 95: e5031, doi: 10.1097/MD.0000000000005031.

10. Zanotti-Cavazzoni SL, Hollenberg SM. Cardiac dysfunction in severe sepsis and septic shock. Curr Opin Crit Care 2009; 15: 392-397, doi: 10.1097/MCC.0b013e3283 $307 a 4$ e. 
11. Otto GP, Sossdorf M, Claus RA, Rodel J, Menge K, Reinhart $\mathrm{K}$, et al. The late phase of sepsis is characterized by an increased microbiological burden and death rate. Crit Care 2011; 15: R183, doi: 10.1186/cc10332.

12. Pierrakos C, Vincent JL. Sepsis biomarkers: a review. Crit Care 2010; 14: R15, doi: 10.1186/cc8872.

13. Bloos F, Reinhart K. Rapid diagnosis of sepsis. Virulence 2014; 5: 154-160, doi: 10.4161/viru.27393.

14. Shen M, Cai L, Jiang $K, X u$ W, Chen $Y, X u Z$. The therapeutic role of inhibition of miR-328 on pulmonary carcinoma induced by chlamydia pneumoniae through targeting histone H2AX. Cancer Biomark 2018, doi: 10.3233/CBM-18 1999.

15. Zhang M, Zhang J, Zhou Q. Elevated expression of microRNA-328-3p suppresses aggressive malignant behaviors via targeting matrix metalloprotease 16 in osteosarcoma. Onco Targets Ther 2019; 12: 2063-2070, doi: 10.2147/OTT.S195022.

16. Lin $\mathrm{CH}$, Chiang $\mathrm{MC}$, Chen YJ. MicroRNA-328 inhibits migration and epithelial-mesenchymal transition by targeting CD44 in nasopharyngeal carcinoma cells. Onco Targets Ther 2018; 11: 2375-2385, doi: 10.2147/OTT.S151665.

17. He F, Lv P, Zhao X, Wang X, Ma X, Meng W, et al. Predictive value of circulating miR-328 and miR-134 for acute myocardial infarction. Mol Cell Biochem 2014; 394: 137144, doi: 10.1007/s11010-014-2089-0.

18. Eiring AM, Harb JG, Neviani P, Garton C, Oaks JJ, Spizzo R, et al. miR-328 functions as an RNA decoy to modulate hnRNP E2 regulation of mRNA translation in leukemic blasts. Cell 2010; 140: 652-665, doi: 10.1016/j.cell.2010.01.007.

19. Zhao D, Li C, Yan H, Li T, Qian M, Zheng N, et al. Cardiomyocyte derived miR-328 Promotes cardiac fibrosis by paracrinely regulating adjacent fibroblasts. Cell Physiol Biochem 2018; 46: 1555-1565, doi: 10.1159/000489201.

20. Du W, Liang H, Gao X, Li X, Zhang Y, Pan Z, et al. MicroRNA-328, a potential anti-fibrotic target in cardiac interstitial fibrosis. Cell Physiol Biochem 2016; 39: 827-836, doi: $10.1159 / 000447793$.

21. Zhang $X$, Xin G, Sun D. Serum exosomal miR-328, miR575 , miR-134 and miR-671-5p as potential biomarkers for the diagnosis of Kawasaki disease and the prediction of therapeutic outcomes of intravenous immunoglobulin therapy. Exp Ther Med 2018; 16: 2420-2432, doi: 10.3892/etm. 2018.6458.

22. Bone RC, Balk RA, Cerra FB, Dellinger RP, Fein AM, Knaus $W A$, et al. Definitions for sepsis and organ failure and guidelines for the use of innovative therapies in sepsis. The ACCP/SCCM Consensus Conference Committee. American College of Chest Physicians/Society of Critical Care Medicine. Chest 1992; 101: 1644-1655, doi: 10.1378/chest.101. 6.1644 .

23. Vincent JL, Moreno R, Takala J, Willatts S, De Mendonca A, Bruining $\mathrm{H}$, et al. The SOFA (Sepsis-related Organ Failure Assessment) score to describe organ dysfunction/failure. On behalf of the Working Group on Sepsis-Related Problems of the European Society of Intensive Care Medicine. Intensive Care Med 1996; 22: 707-710, doi: 10.1007/BF01709751.

24. Knaus WA, Draper EA, Wagner DP, Zimmerman JE. APACHE II: a severity of disease classification system. Crit Care Med 1985; 13: 818-829, doi: 10.1097/00003246-1985 10000-00009.
25. Dhas BB, Dirisala VR, Bhat BV. Expression levels of candidate circulating microRNAs in early-onset neonatal sepsis compared with healthy newborns. Genomics Insights 2018; 11: 1178631018797079 , doi: $10.1177 / 1178631018797079$.

26. Fabri-Faja N, Calvo-Lozano O, Dey P, Terborg RA, Estevez MC, Belushkin A, et al. Early sepsis diagnosis via protein and miRNA biomarkers using a novel point-of-care photonic biosensor. Anal Chim Acta 2019; 1077: 232-242, doi: 10.1016/j.aca.2019.05.038

27. Xia HL, Lv Y, Xu CW, Fu MC, Zhang T, Yan XM, et al. MiR$513 \mathrm{c}$ suppresses neuroblastoma cell migration, invasion, and proliferation through direct targeting glutaminase (GLS). Cancer Biomark 2017; 20: 589-596, doi: 10.3233/CBM170577.

28. Huang F, Wang B, Zeng J, Sang S, Lei J, Lu Y. MicroRNA$374 \mathrm{~b}$ inhibits liver cancer progression via down regulating programmed cell death-1 expression on cytokine-induced killer cells. Oncol Lett 2018; 15: 4797-4804, doi: 10.3892/ ol.2018.7951.

29. Benz F, Roy S, Trautwein C, Roderburg C, Luedde T. Circulating MicroRNAs as biomarkers for sepsis. Int $\mathrm{J} \mathrm{Mol}$ Sci 2016; 17. pii:E78, doi: 10.3390/ijms17010078.

30. Liu Y, Cheng Z, Pan F, Yan W. MicroRNA-373 promotes growth and cellular invasion in osteosarcoma cells by activation of the PI3K/AKT-Rac1-JNK pathway: the potential role in spinal osteosarcoma. Oncol Res 2017; 25: 989-999, doi: 10.3727/096504016X14813867762123.

31. Zhang X, Ai F, Li X, Tian L, Wang X, Shen S, et al. MicroRNA-34a suppresses colorectal cancer metastasis by regulating notch signaling. Oncol Lett 2017; 14: 2325-2333, doi: 10.3892/ol.2017.6444.

32. Yuan J, Zheng Z, Zheng Y, Lu X, Xu L, Lin L. microRNA-328 is a favorable prognostic marker in human glioma via suppressing invasive and proliferative phenotypes of malignant cells. Int J Neurosci 2016; 126: 145-153, doi: 10.3109/ 00207454.2014 .1002610$.

33. Ulivi P, Foschi G, Mengozzi M, Scarpi E, Silvestrini R, Amadori $D$, et al. Peripheral blood miR-328 expression as a potential biomarker for the early diagnosis of NSCLC. Int $\mathrm{J} \mathrm{Mol} \mathrm{Sci}$ 2013; 14: 10332-10342, doi: 10.3390/ijms140510332.

34. Lv X, Wang $\mathrm{H}$. Pathophysiology of sepsis-induced myocardial dysfunction. Mil Med Res 2016; 3: 30, doi: 10.1186/ s40779-016-0099-9.

35. Chen H, Wang X, Yan X, Cheng X, He X, Zheng W. LncRNA MALAT1 regulates sepsis-induced cardiac inflammation and dysfunction via interaction with miR-125b and p38 MAPK/ NFkappaB. Int Immunopharmacol 2018; 55: 69-76, doi: 10.1016/j.intimp.2017.11.038.

36. Chen S, Hua F, Lu J, Jiang Y, Tang Y, Tao L, et al. Effect of dexmedetomidine on myocardial ischemia-reperfusion injury. Int J Clin Exp Med 2015; 8: 21166-21172.

37. Pathan N, Franklin JL, Eleftherohorinou H, Wright VJ, Hemingway CA, Waddell SJ, et al. Myocardial depressant effects of interleukin 6 in meningococcal sepsis are regulated by p38 mitogen-activated protein kinase. Crit Care Med 2011; 39: 1692-1711, doi: 10.1097/CCM.0b013e3182186d27.

38. Zhang Y, Roth JA, Yu H, Ye Y, Xie K, Zhao H, et al. A 5microRNA signature identified from serum microRNA profiling predicts survival in patients with advanced stage nonsmall cell lung cancer. Carcinogenesis 2019; 40: 643-650, doi: 10.1093/carcin/bgy132. 